JOURNAL OF THE

CHUNGCHEONG MATHEMATICAL SOCIETY

Volume 27, No. 4, November 2014

http://dx.doi.org/10.14403/jcms.2014.27.4.563

\title{
CURVES OF MAXIMAL GENUS ON SURFACE SCROLLS
}

\author{
HEESANG PARK*
}

\begin{abstract}
We investigate a minimal set of generators of a homogeneous ideal of a curve of degree $d$ on a linearly normal smooth surface scroll $W$ in $\mathbf{P}^{r}$ whose arithmetic genus is maximal among curves of degree $d$ on $W$.
\end{abstract}

\section{Introduction}

For given two integers $d, r$ with $d \geq r \geq 3$, Castelnuovo [2] proved that there is an upper bound $\pi(d, r)$ for the arithmetic genus of irreducible, nondegenerate curves of degree $d$ in $\mathbf{P}^{r}$. Here $\pi(d, r)$ is given by

$$
\pi(d, r)=\left(\begin{array}{c}
m \\
2
\end{array}\right)(r-1)+m \epsilon
$$

where $d=m(r-1)+\epsilon+l, \epsilon=0, \ldots, r-2$. He also classified curves for which the bound is attained.

In this paper we will investigate a curve lying on a linearly normal smooth surface scroll $W$ in $\mathbf{P}^{r}$ whose arithmetic genera are maximal among curves of degree $d$ on $W$; Theorem 2.3. Especially we are interested in a minimal set of generators of homogeneous ideals of such curves; Proposition 2.5.

Every linearly normal surface scroll in $\mathbf{P}^{r}$ is the image of the unique ruled surface by an embedding defined by a unisecant linear series on the ruled surface. So a degree of a curve on $W$ is given by a fixed ample divisor $H$ on the ruled surface which gives the embedding of the ruled surface into $\mathbf{P}^{r}$. So the paper deals with a curve on a ruled surface with a fixed ample divisor on the ruled surface.

Received June 09, 2014; Accepted October 20, 2014.

2010 Mathematics Subject Classification: Primary 14 H55.

Key words and phrases: curve, maximal genus, surface scroll. 


\section{Generators of homogeneous ideals of maximal curves}

Let $Y$ be a smooth curve of genus $g_{y} \geq 1$. Let $\pi: \mathbf{P}(\mathcal{E}) \rightarrow Y$ be a ruled surface over $Y$ with the $e$-invariant $e$ and let $S_{0}$ be a minimal degree section of $\mathbf{P}(\mathcal{E})$, that is, $S_{0}$ is the section of $\mathbf{P}(\mathcal{E})$ with $S_{0}^{2}=-e$. Assume that $\mathcal{E}_{0}=\mathcal{E} \otimes \mathcal{O}_{Y}(-N)$ is normalized. Setting $n=\operatorname{deg} N$ and $\mathcal{O}_{Y}(B)=\operatorname{det} \mathcal{E}$ with $b=\operatorname{deg} B$, we have

$$
e=-\operatorname{deg} \mathcal{E}_{0}=2 n-b .
$$

Throughout this paper, fix a divisor $Z \in \operatorname{Div}(Y)$ with

$$
z:=\operatorname{deg} Z \geq \max \left\{2 g_{y}+1,2 g_{y}+1+e\right\} \text {. }
$$

and set

$$
H=S_{0}+\pi^{*} Z \text { and } r=\operatorname{dim}|H| .
$$

The $H$-degree of a curve $X \subset \mathbf{P}(\mathcal{E})$ is defined by the intersection number $X . H$.

Lemma 2.1. The linear series $|H|$ on $\mathbf{P}(\mathcal{E})$ is very ample and

$$
r=\operatorname{dim}|H|=-e+2 z-2 g_{y}+1 .
$$

Proof. By [3, V, Ex. 2.11], the linear series $|H|$ is very ample. We now count $h^{0}(\mathbf{P}(\mathcal{E}), H)$. Since $\pi_{*}\left(\mathcal{O}_{\mathbf{P}(\mathcal{E})}\left(S_{0}\right)\right) \cong \mathcal{E}_{0}$ by [3, V, 2.4], it follows by the projection formula that

$$
\begin{aligned}
h^{0}(\mathbf{P}(\mathcal{E}), H) & =h^{0}\left(Y, \mathcal{E}_{0} \otimes \mathcal{O}_{Y}(Z)\right) \\
& =\operatorname{deg}\left(\mathcal{E}_{0} \otimes \mathcal{O}_{Y}(Z)\right)-2\left(g_{y}-1\right)+h^{1}\left(Y, \mathcal{E}_{0} \otimes \mathcal{O}_{Y}(Z)\right) \\
& =-e+2 z-2 g_{y}+2+h^{0}\left(Y, \mathcal{E}_{0}^{\vee} \otimes \mathcal{O}_{Y}\left(K_{Y}-Z\right)\right) .
\end{aligned}
$$

It is enough to prove that $h^{0}\left(Y, \mathcal{E}_{0}^{\vee} \otimes \mathcal{O}_{Y}\left(K_{Y}-Z\right)\right)=0$. Note that we have

$\mathcal{E}_{0}^{\vee} \cong \mathcal{E}_{0} \otimes \operatorname{det} \mathcal{E}_{0}{ }^{-1} \cong \mathcal{E}_{0} \otimes\left(\operatorname{det} \mathcal{E}^{-1} \otimes \mathcal{O}_{Y}(2 N)\right)=\mathcal{E}_{0} \otimes \mathcal{O}_{Y}(-B+2 N) ;$

hence, it follows that

$$
h^{0}\left(Y, \mathcal{E}_{0}^{\vee} \otimes \mathcal{O}_{Y}\left(K_{Y}-Z\right)\right)=h^{0}\left(Y, \mathcal{E}_{0} \otimes \mathcal{O}_{Y}\left(K_{Y}-Z-B+2 N\right)\right) .
$$

By Equation (2.1) and the assumption $z-e \geq 2 g_{y}+1$, we have

$$
\operatorname{deg}\left(K_{Y}-Z-B+2 N\right)=2 g_{y}-2-(z-e)<0 ;
$$

however, $\mathcal{E}_{0}$ is normalized, hence

$$
h^{0}\left(Y, \mathcal{E}_{0} \otimes \mathcal{O}_{Y}\left(K_{Y}-Z-B+2 N\right)\right)=0 .
$$


REMARK 2.2. Let

$$
\phi_{H}: \mathbf{P}(\mathcal{E}) \hookrightarrow \mathbf{P}^{r}
$$

be an embedding defined by $|H|$ and set

$$
W=\phi_{H}(\mathbf{P}(\mathcal{E})) \text {. }
$$

Since $H^{2}=-e+2 z$, the surface scroll $W$ is of degree $\left(r-1+2 g_{y}\right)$ in $\mathbf{P}^{r}$.

Theorem 2.3. The maximal arithmetic genus of curves of degree $d$ lying on $W=\phi_{H}(\mathbf{P}(\mathcal{E}))$ in $\mathbf{P}^{r}$ is equal to

$$
\left(\begin{array}{c}
m \\
2
\end{array}\right)\left(r-1+2 g_{y}\right)+m \varepsilon+g_{y}
$$

where

$$
m=\left[\frac{d-1+g_{y}}{r-1+2 g_{y}}\right]
$$

is the greatest integer not exceeding $\left(d-1+g_{y}\right) /\left(r-1+2 g_{y}\right)$ and

$$
\varepsilon=\left(d-1+g_{y}\right)-\left(r-1+2 g_{y}\right) m .
$$

Proof. Let $X$ be a curve on $\mathbf{P}(\mathcal{E})$ of $H$-degree $d$. Set $X \sim i S_{0}+\pi^{*} J$ for some $J \in \operatorname{Div}(Y)$. We have

$$
d=X . H=-i e+i z+j,
$$

where $j=\operatorname{deg} J$. By the adjunction formula, we get

$$
p_{a}(X)=(i-1)\left(d-1+g_{y}-\frac{i}{2}\left(r-1+2 g_{y}\right)\right)+g_{y} .
$$

An elementary calculation shows that $p_{a}(X)$ is maximized for fixed $d$ and $r$ when

$$
i=\left[\frac{d-1+g_{y}}{r-1+2 g_{y}}\right]+1
$$

Remark 2.4. If $X \subset \mathbf{P}(\mathcal{E})$ is a curve of $H$-degree $d$ with

$$
p_{a}(X)=\left(\begin{array}{c}
m \\
2
\end{array}\right)\left(r-1+2 g_{y}\right)+m \varepsilon+g_{y},
$$

then we have

$$
X \sim(m+1) H+\pi^{*}(J-(m+1) Z) .
$$

Note that

$$
\operatorname{deg}(J-(m+1) Z)=-r+\varepsilon+2-3 g_{y} .
$$


Proposition 2.5. Assume that $z \geq 3+e$ if $g_{y}=1$ and $z \geq \max \left\{3 g_{y}+\right.$ $\left.e, 3 g_{y}+\frac{e}{2}\right\}$ if $g_{y} \geq 2$. Let $X \subset \mathbf{P}(\mathcal{E})$ be a curve of $H$-degree $d$ with the maximal arithmetic genus

$$
p_{a}(X)=\left(\begin{array}{c}
m \\
2
\end{array}\right)\left(r-1+2 g_{y}\right)+m \varepsilon .
$$

Let $I \subset \mathbb{C}\left[X_{0}, \ldots, X_{r}\right]$ be the homogeneous ideal defining $X$ under the embedding by $|H|$. If $|(m+1) Z-J|$ is base-point-free, then a minimal set of generators for $I$ consists of quadrics and polynomials of degree $m+1$. On the other hand, if $|(m+1) Z-J|$ has a base point, then a minimal set of generators for I consists of quadrics, polynomials of degree $m+1$, and, in addition, polynomials of degree $m+2$.

REMARK 2.6. By [4] and the assumption on the degree $z$, the very ample linear series $|H|$ satisfies $N_{1}$ property, that is, the embedding $\phi_{H}$ is a projectively normal embedding and the homogeneous ideal of $W$ is generated by quadrics.

We divide the proof of Proposition 2.5 into the following three lemmas.

Lemma 2.7. Every hypersurface of degree $l \leq m$ containing $X$ contains $W$.

Proof. Consider the short exact sequence of ideal sheaves

$$
0 \rightarrow \mathcal{I}_{W, \mathbf{P}^{r}}(l) \rightarrow \mathcal{I}_{X, \mathbf{P}^{r}}(l) \rightarrow \mathcal{I}_{X, W}(l) \rightarrow 0 .
$$

Since $l<m+1$, we have

$$
\begin{aligned}
H^{0}\left(W, \mathcal{I}_{X, W}(l)\right) & =H^{0}(\mathbf{P}(\mathcal{E}),-X+l H) \\
& =H^{0}\left(\mathbf{P}(\mathcal{E}),(l-m-1) S_{0}+\pi^{*}(l Z-J)\right)=0 .
\end{aligned}
$$

Therefore

$$
H^{0}\left(\mathbf{P}^{r}, \mathcal{I}_{X, \mathbf{P}^{r}}(l)\right)=H^{0}\left(\mathbf{P}^{r}, \mathcal{I}_{W, \mathbf{P}^{r}}(l)\right)
$$

for $l \leq m$.

Lemma 2.8. Under the hypothesis of Proposition 2.5, modulo the ideal of $W$, there are exactly $h^{0}(Y,(m+1) Z-J)$ linearly independent hypersurfaces of degree $m+1$ containing $X$.

Proof. By Remark 2.6, we have

$$
H^{1}\left(\mathbf{P}^{r}, \mathcal{I}_{W, \mathbf{P}^{r}}(m+1)\right)=0 .
$$

From the short exact sequence of ideal sheaves, we have 


$$
\begin{aligned}
0 & \rightarrow H^{0}\left(\mathbf{P}^{r}, \mathcal{I}_{W, \mathbf{P}^{r}}(m+1)\right) \rightarrow H^{0}\left(\mathbf{P}^{r}, \mathcal{I}_{X, \mathbf{P}^{r}}(m+1)\right) \\
& \rightarrow H^{0}\left(W, \mathcal{I}_{X, W}(m+1)\right) \rightarrow 0
\end{aligned}
$$

Since

$$
H^{0}\left(W, \mathcal{I}_{X, W}(m+1)\right) \cong H^{0}(Y,(m+1) Z-J),
$$

the proof is done.

Lemma 2.9. Under the hypothesis of Proposition 2.5, the natural map

$$
H^{0}\left(W, \mathcal{I}_{X, W}(m+2)\right) \otimes H^{0}\left(W, \mathcal{O}_{W}(\alpha)\right) \rightarrow H^{0}\left(W, \mathcal{I}_{X, W}(m+2+\alpha)\right)
$$

is surjective for any $\alpha>0$. Furthermore, if $|(m+1) Z-J|$ is base-pointfree, then the natural map

$$
H^{0}\left(W, \mathcal{I}_{X, W}(m+1)\right) \otimes H^{0}\left(W, \mathcal{O}_{W}(\alpha)\right) \rightarrow H^{0}\left(W, \mathcal{I}_{X, W}(m+1+\alpha)\right)
$$

is also surjective for any $\alpha>0$.

Proof. Since

$$
\mathcal{I}_{X, W}(m+2) \cong \mathcal{O}_{\mathbf{P}(\mathcal{E})}\left(S_{0}+\pi^{*}((m+2) Z-J)\right),
$$

we have to prove that the map

$$
\begin{aligned}
H^{0}\left(\mathbf{P}(\mathcal{E}), S_{0}+\right. & \left.\pi^{*}((m+2) Z-J)\right) \otimes H^{0}\left(\mathbf{P}(\mathcal{E}), \alpha S_{0}+\pi^{*}(\alpha Z)\right) \\
& \longrightarrow H^{0}\left(\mathbf{P}(\mathcal{E}),(\alpha+1) S_{0}+\pi^{*}((m+2) Z-J+\alpha Z)\right)
\end{aligned}
$$

is surjective. Hence, it is equivalent to prove that the following map is surjective:

$$
\begin{aligned}
H^{0}\left(Y, \mathcal{E}_{0} \otimes \mathcal{O}_{Y}(\right. & (m+2) Z-J)) \otimes H^{0}\left(Y, \operatorname{sym}^{\alpha} \mathcal{E}_{0} \otimes \mathcal{O}_{Y}(\alpha Z)\right) \\
& \longrightarrow H^{0}\left(Y, \mathcal{E}_{0} \otimes \operatorname{sym}^{\alpha} \mathcal{E}_{0} \otimes \mathcal{O}_{Y}((m+2) Z-J+\alpha Z)\right) .
\end{aligned}
$$

For this, we have the following result:

Lemma 2.10 ([1, Proposition 2.2]). Let $\mathcal{F}$ and $\mathcal{G}$ be vector bundles over $Y$ with $\mathcal{F}$ generated by global sections. Let $\mu^{-}(\mathcal{L}):=\min \{\mu(\mathcal{Q})$ : $\mathcal{L} \rightarrow \mathcal{Q} \rightarrow 0\}$, where $\mu(\mathcal{Q})=\operatorname{deg} \mathcal{Q} / \operatorname{rank} \mathcal{Q}$. If

1. $\mu^{-}(\mathcal{G})>2 g_{y}$ and

2. $\mu^{-}(\mathcal{G})>2 g_{y}+\operatorname{rank}(\mathcal{F})\left(2 g_{y}-\mu^{-}(\mathcal{F})\right)-2 h^{1}(Y, \mathcal{F})$,

then the natural multiplication map

$$
\tau: H^{0}(Y, \mathcal{F}) \otimes H^{0}(Y, \mathcal{G}) \rightarrow H^{0}(Y, \mathcal{F} \otimes \mathcal{G}),
$$

is surjective. 
Set $\mathcal{F}=\mathcal{E}_{0} \otimes \mathcal{O}_{Y}((m+2) Z-J)$ and $\mathcal{G}=\operatorname{sym}^{\alpha} \mathcal{E}_{0} \otimes \mathcal{O}_{Y}(\alpha Z)$. First, we will show that $\mathcal{F}$ is generated by global sections. By [1, Lemma 1.12], a vector bundle $\mathcal{L}$ on $Y$ is generated by global sections if $\mu^{-}(\mathcal{L}) \geq 2 g_{y}$; hence it is enough to show that

$$
\mu^{-}(\mathcal{F}) \geq 2 g_{y} .
$$

Note that $\mu^{-}\left(\mathcal{E}_{0}\right)=-e$ if $e \geq 0$ and $\mu^{-}\left(\mathcal{E}_{0}\right)=-\frac{e}{2}$ if $e<0$; cf. [4, §2.4]. From $\left[1\right.$, Lemma 2.5], we have $\mu^{-}(\mathcal{L} \otimes \mathcal{M})=\mu^{-}(\mathcal{L})+\mu^{-}(\mathcal{M})$ for vector bundles $\mathcal{L}$ and $\mathcal{M}$ on $Y$. Therefore we have

$$
\begin{aligned}
& \mu^{-}(\mathcal{F})=\mu^{-}\left(\mathcal{E}_{0}\right)+\mu^{-}\left(\mathcal{O}_{Y}((m+2) Z-J)\right) \\
& =\left\{\begin{array}{ll}
-e+r-\varepsilon-2+3 g_{y}+z & \text { if } e \geq 0, \\
-\frac{e}{2}+r-\varepsilon-2+3 g_{y}+z & \text { if } e<0
\end{array} \quad\right. \text { by Equation (2.6) } \\
& \geq\left\{\begin{array}{ll}
-e+g_{y}+z & \text { if } e \geq 0, \\
-\frac{e}{2}+g_{y}+z & \text { if } e<0
\end{array} \quad \text { since } \varepsilon \leq r-2+2 g_{y}\right. \\
& \geq 2 g_{y} \text {. } \quad \text { by the assumption on } z
\end{aligned}
$$

Second, we will prove that $\mu^{-}(\mathcal{G})>2 g_{y}$. From [1, Lemma 2.5], we have $\mu^{-}\left(\operatorname{sym}^{\alpha} \mathcal{E}_{0}\right)=\alpha \mu^{-}\left(\mathcal{E}_{0}\right)$. Therefore we have

$$
\begin{aligned}
\mu^{-}(\mathcal{G}) & =\alpha \mu^{-}\left(\mathcal{E}_{0}\right)+\mu^{-}\left(\mathcal{O}_{Y}(\alpha Z)\right) \\
& = \begin{cases}-\alpha e+\alpha z & \text { if } e \geq 0, \\
-\alpha \cdot \frac{e}{2}+\alpha z & \text { it } e \leq-1\end{cases} \\
& >2 g_{y} .
\end{aligned}
$$

Finally, since $2 g_{y}-\mu^{-}(\mathcal{F}) \leq 0$, the condition (2) of Lemma 2.10 holds. So fat, we proved the first assertion of Lemma 2.9.

For the second assertion, we have

$$
\mathcal{I}_{X, W}(m+1) \cong \mathcal{O}_{\mathbf{P}(\mathcal{E})}\left(\pi^{*}((m+1) Z-J)\right) ;
$$

hence it is equivalent to prove that the following map is surjective:

$$
\begin{aligned}
H^{0}(Y,(m+1) Z-J) & \otimes H^{0}\left(Y, \operatorname{sym}^{\alpha} \mathcal{E}_{0} \otimes \mathcal{O}_{Y}(\alpha Z)\right) \\
\longrightarrow & H^{0}\left(Y, \operatorname{sym}^{\alpha} \mathcal{E}_{0} \otimes \mathcal{O}_{Y}((m+1) Z-J+\alpha Z)\right) .
\end{aligned}
$$

Set $\mathcal{F}=\mathcal{O}_{Y}((m+1) Z-J)$ and $\mathcal{G}=\operatorname{sym}^{\alpha} \mathcal{E}_{0} \otimes \mathcal{O}_{Y}(\alpha Z)$. Since we assumed that $\mathcal{F}$ is generated by global sections, the second assertion also follows by Lemma 2.10 . 
REMARK 2.11. It is clear that the natural map

$$
H^{0}\left(W, \mathcal{I}_{X, W}(m+1)\right) \otimes H^{0}(W, \alpha H) \rightarrow H^{0}\left(W, \mathcal{I}_{X, W}(m+1+\alpha)\right)
$$

cannot be surjective if $|(m+1) Z-J|$ has a base point.

\section{References}

[1] D. C. Butler, Normal generation of vector bundles over a curve, J. Differential Geom. 39 (1994), no. 1, 1-34.

[2] G. Castelnuovo, Ricerche di geometria sulle curve algebriche, Ani R. Accad. Sci. Torino 24 (1889)

[3] R. Hartshorne, Algebraic geometry, Springer-Verlag, 1977, Graduate Texts in Mathematics, No. 52.

[4] E. Park, On higher syzygies of ruled surfaces, Trans. Amer. Math. Soc. 358 (2006), no. 8, 3733-3749.

Department of Mathematics

Konkuk University

Seoul 143-701, Republic of Korea

E-mail: HeesangPark@konkuk.ac.kr 